\title{
ESTABELECIMENTO E RECUPERAÇÃO DO PARASITÓIDE Ageniaspis citricola LOGVINOVSKAYA (HYM.:ENCYRTIDAE), SOBRE A LARVA MINADORA DOS CITROS, Phillocnistis citrella STAINTON (LEP.: GRACILLARIIDAE) EM CONDI- COOEES DE CAMPO
}

Sônia Maria N.M.Montes ${ }^{1}$

Geraldo Papa ${ }^{2}$

Leonardo Coutinho Cerâvolo ${ }^{1}$ Antonio Carlos Rossi ${ }^{1}$ Aparecida Conceição Boliani²

\section{RESUMO}

A vespa Ageniaspis citricola é um endoparasitóide exótico, que parasita ovos e larvas de primeiros instares de Phyllocnistis citrella, e é excelente parasitóide da larva minadora dos citros. A larva minadora dos citros é uma das mais sérias pragas da citricultura brasileira, uma vez que provoca lesões nas brotações novas de citros, propiciando a entrada e disseminação da bactéria causadora do cancro cítrico, Xanthomonas axonopodis pv. citri. O objetivo deste trabalho foi avaliar o estabelecimento e a recuperação do parasitóide introduzido $A$. cifricola no controle de $P$. cifrella. As liberações foram realizadas em um pomar comercial no Município de Álvares Machado, SP, região de Presidente Prudente, de 164 plantas de laranja Lima, com 3,5 anos de idade, utilizando-se 4 parcelas de 30 plantas cada, onde, através de uma gaiola confeccionada com $25 \mathrm{~cm}$ de tubo de PVC de 4 polegadas $(10 \mathrm{~cm})$ de diâmetro, realizou-se a liberação do parasitóide. Em cada parcela foram liberados números diferentes de parasitóides: Parcela 1: 30 folhas/gaiola (aproximadamente 90 insetos/gaiola), Parcela 2: 90 folhas/gaiola (aproximadamente 270 inse-

Pólo Regional da Alta Sorocabana-APTA - Rod.Raposo Tavares Km 561- Caixa Postal 298, 19015-970, Presidente Prudente - SP. FEIS/UNESP-Av. Brasil, 56-Centro, 15385000 , Ilha Solteira- SP. 
tos/gaiola) e Parcela 3: 180 folhas/gaiola (aproximadamente 540 insetos/ gaiola). As avaliações foram realizadas aos 23, 40, 58 e 73 dias após a liberação, tomando-se de 10 plantas ao acaso, que apresentavam brotações novas infestadas, 4 ramos novos onde folhas com sintomas do ataque da larva minadora foram mantidas em BOD a $25+20$ C U.R. $70 \%$ e fotofase de 14 horas, até a emergência de parasitóides nativos e A. citricola.. Ao longo das 4 avaliações realizadas, observaram-se melhores resultados nos tratamentos 2 e 3 , onde foram liberados aproximadamente 270 e 540 insetos/gaiola, alcançando índices de $86,7 \%$ e $85,7 \%$ de larvas minadora parasitadas, respectivamente.

Palavras-chave: larva minadora, citros, parasitóides, liberação.

\section{ABSTRACT}

ESTABLISHMENT AND RECUPERATION OF THE

PARASITOIDS Ageniaspis citricola LOGVINOVSKAYA (HYMENOPTERA:ENCYRTIDAE), ON THE Phyllocnistis citrella STAINTON (LEPIDOPTERA: GRACILLARIIDAE), IN ORANGE ORCHARD

The objective of this work was to evaluate the establishment and recuperation of the introduced parasitoid, Ageniaspis citricola, in the control of the citrus leafminer, Phyllocnistis citrella. The experiment was carried out at a commercial orchard in the Municipal district of Álvares Machado/SP; 164 citrus plants variety Lima, with 3.5 years of age were used. The area was divided in 4 plots of 30 plants each. In each plot were released, through a cage made with $25 \mathrm{~cm}$ of a 4 inch diameter PVC tube, 90,270 and 540 adult parasitoids of $A$. citricola. The evaluations were accomplished 23, 40, 58 and 73 days after the liberation, on 10 plants of each plot. Of these plants, 4 new branches were taken, containing symptoms of attack of citrus leafminer. The leaves of these branches were maintained in controlled conditions, with temperature of $25+20 \mathrm{C}$ and photofase of 14 hours, until emergency of native parasitoids and the introduced parasitoid A. citricola. After 4 evaluations, the best results 
were observed in the plots where 270 and 540 insects were liberated, reaching indexes of $86,7 \%$ and $85,7 \%$ of parasitism, respectively.

Key words: leafminer, citrus, parasitoids, liberation.

\section{INTRODUÇÃO}

A larva minadora dos citros, Phyllocnistis citrella Stainton, é uma séria praga dos citros e de espécies de plantas da família das Rutáceas (Heppner, 1993). No Brasil foi identificada pela primeira vez em março de 1996, na região de Limeira, Estado de São Paulo, e tomou-se uma das maiores pragas da citricultura brasileira. Dois meses após a primeira constatação havia se disseminado por todo o Estado, e dois anos após encontrava-se nos Estados do Amazonas, Rondônia, Roraima, Piauí, Bahia, Mato Grosso do Sul, Goiás, Minas Gerais, Rio de Janeiro, Paraná e Tocantins (Nascimento \& Vidal, 1998).

As lagartas de $P$. citrella atacam as folhas novas de plantas cítricas, minando, preferencialmente suas superficies inferiores (Chagas et al., 1998). Constroem minas típicas, com o tecido foliar assumindo coloração prateada, e tomam-se retorcidas, ocorrendo morte do tecido foliar e queda prematura das folhas (Knapp et al., 1995).

Embora possa causar danos diretos, a importância maior desta praga é sua relação com o cancro cítrico. As lesões que faz nas folhas, sobretudo nas novas, servem de porta de entrada para a bactéria Xanthomonas axonopodis pv. citri, causadora da doença, o que facilitou a disseminação do cancro cítrico (Fundecitrus, 2001).

Os adultos de $P$. curella vivem geralmente 2 a 10 dias. Sua atividade ocorre ao anoitecer ou nas primeiras horas da madrugada (Knapp et al., 1995). A eclosão das larvas ocorre 2 a 10 dias após a postura, podendo uma fêmea ovipositar 20 ovos/noite para mais de 50, em uma vida média de 5 a 20 dias (Smith \& Hoy, 1995).

O estágio pupal pode durar de 6 a 22 dias, dependendo da época do ano, de maneira que uma geração é completada em 14 a 17 dias, nos meses mais quentes de verão, e em 52 dias, no inverno (Smith \& Hoy, 1995). 
O controle químico da larva tem-se mostrado difícil por fatores como incapacidade do produto em penetrar a cutícula da folha e atingir a larva ou pupa, limitada atividade residual do produto e elevada freqüencia de brotações pela planta (Knapp et al.., 1995).

Em vários países, onde a praga ocorre, o controle biológico tem sido adotado como componente de elevada prioridade em programas de manejo integrado de pragas. Nos EUA, no Estado da Flórida, liberações do parasitóide introduzido $A$. citricola, proporcionaram taxa de parasitismo de $99 \%$ após 15 dias de liberação (Smith \& Hoy, 1995).

Parasitóides nativos também têm exercido importante ação no controle da praga, com uma taxa de parasitismo de $66 \%$ apontada por Browning \& Pefta (1995) na Flórida pelas espécies Pnigalio sp., Closterocerus cinctipinnus, Horismenus sp., Elasmus tischeriae, Sympiesis sp e Zagrammosoma multilineatumn. No Brasil já foram identificados Galeopsomyia sp., Cirrospilus sp., Elasmus sp e vespas predadoras (Gravena, 1998). Penteado-Dias et al., 1997, relataram ainda os gêneros Pachyneuron (Pteromalidae) e Telenomus (Scelionidae) parasitando larvas de $P$. citrella.

Estudos realizados por Paiva et al., 1998, mostram que Galeopsomyia sp. é o principal parasitóide do minador dos citros no Brasil, seguido por Cirrospilus sp. (Gravena, 1998), sendo que Galeopsomyia fausta se desenvolve externamente em larvas de 30 instar, pré-pupa e pupa, e foi observada em 40 e $70 \%$ das câmaras pupais (Paiva et al., 1998).

O Brasil num esforço conjunto de pesquisadores do Fundecitrus, Esalq/USP, Embrapa - Meio Ambiente e Gravena - Manejo Ecológico de Pragas Ltda., importou, em junho de 1998, a vespa Ageniaspis citricola, para criação e liberação no Estado de São Paulo, tendo em vista a baixa taxa de parasitismo exercido pelos parasitóides nativos, e tendo em vista experiência de outros países que importaram o parasitóide.

Em outubro de 1998, foi feita a primeira soltura pela ação conjunta da Gravena e Esalq de cerca de 400 vespinhas na Fazenda Santo Antônio, município de Taquaritinga, SP, e no dia 19/11 foram constatados descendentes no pomar. No final de 1999, o parasitóide já era encon- 
trado em pomares onde não se fez sua liberação, indicando que houve rápida disseminação. Em 2000 continuaram as liberações em pomares, verificando-se até 1000\% de eficiência do parasitóide em brotações novas (Gravena, 2000). Em São Paulo, o parasitóide foi liberado em cerca de 200 pomares de 60 municípios, mas já é encontrado em todo o Estado, devido à grande capacidade de dispersão que possui, tendo sido detectado a $40 \mathrm{~km}$ de distância do local de soltura em algumas propriedades. Tornou-se um inimigo natural importante no meio ambiente brasileiro, baixando em até $90 \%$, em alguns pomares, a população da praga (Fundecitrus, 2001).

A. citricola é um endoparasitóide de ovos e larvas de primeiros instares de $P$. citrella, produzindo de 1 a 10 individuos/hospedeiro. É eficiente parasito da larva minadora dos citros (Smith \& Hoy, 1995); pode ser afetado pelo uso de inseticidas não seletivos na cultura.

No presente estudo realizaram-se liberações do parasitóide introduzido $A$. citricola, com o objetivo de avaliar o seu estabelecimento e recuperação sobre a larva minadora dos citros $P$. citrella.

\section{MATERIAL E MÉTODOS}

O experimento para estudo de liberação do parasitóide Ageniaspis citricola Logvinoskaya, 1983, foi realizado em pomar comercial, localizado no município de Alvares Machado, região de Presidente Prudente, SP. Os parasitóides foram criados e cedidos pela Gravena - Manejo Ecológico de Pragas Ltda, Jaboticabal, SP e Esalq/USP- Departamento de Entomologia, Fitopatologia e Zoologia Agrícola, Piracicaba, SP.

Folhas de limão cravo (Citrus limonia Osbeck) com pupas do parasitóide foram acondicionadas em caixas plásticas forradas com papel de filtro umedecidas com água esterilizada, com uma abertura na tampa revestida de náilon para permitir trocas gasosas. Estas caixas foram colocadas em caixas de isopor com gelo para o transporte dos parasitóides.

A liberação foi realizada em um pomar de 164 plantas de laranja Lima (Citrus sinensis Osbeck), enxertadas sobre o porta-enxerto limão Cravo, com 3,5 anos de idade, plantadas em espaçamento de $4 \times 2 \mathrm{~m}$, 
localizado no município de Alvares Machado, SP, região de Presidente Prudente.

Para a liberação do parasitóide $A$. citricola , utilizaram-se gaiolas confeccionadas com $25 \mathrm{~cm}$ de tubo de PVC de 4 polegadas de diâmetro, vedadas com tela tipo náilon nas extremidades. Cada gaiola continha uma quantidade variável de folhas de limão Cravo com larvas de $P$. citrella parasitadas pelo parasitóide $A$. citricola com aproximadamente 14 dias de parasitismo, conforme o tratamento. As folhas foram colocadas sobre papel de filtro umedecido com água esterilizada para evitar seu ressecamento.

Utilizaram-se 4 parcelas de 30 plantas cada, sendo a gaiola instalada em uma planta, no centro de cada talhão do tratamento, por um período de 3 dias.

Em cada parcela foram liberados números diferentes de parasitóides: Parcela 1: 30 folhas/gaiola (aproximadamente 90 insetos/ gaiola); Parcela 2: 90 folhas/gaiola (aproximadamente 270 insetos/gaiola); Parcela 3: 180 folhas/gaiola (aproximadamente 540 insetos/gaiola).

As avaliações foram realizadas aos $23,40,58$ e 73 dias após a liberação, com 10 plantas que apresentavam brotações novas infestadas, ao se caminhar em espiral dentro do talhão. Em cada planta foram tomadas, aleatoriamente, 4 ramos novos, onde se observou o número de folhas de cada ramo e o número total de folhas com sintoma recente do ataque de $P$. citrella. As folhas com sintomas de ataque da larva minadora foram enviadas ao laboratório, acondicionadas em placas de Petri e mantidas em câmara de climatização à temperatura de $25 \pm 20 \mathrm{C}$, UR 70 $\pm 10 \%$ e fotofase de 14 horas, até a emergência de parasitóides nativos e A. citricola.

Os parasitóides nativos coletados foram mantidos em álcool $70 \%$ para posterior identificação na Universidade Federal de São Carlos. Os dados obtidos nas avaliações foram acumulados e processados através da fórmula $\mathrm{x}=(\mathrm{y} / \mathrm{n}) .100$, onde $\mathrm{x}$ é o percentual de insetos emergidos, y é o número de insetos emergidos ( $P$. citrella, $A$. citricola e parasitóides nativos) e $\mathbf{n}$ é o número total de folhas com larvas ou pupas parasitadas. 


\section{RESULTADOS E DISCUSSÃO}

Verificou-se que o estabelecimento e a recuperação do parasitóide liberado, $A$. citricola ao longo de quatro avaliações realizadas, mostrou melhores resultados nas parcelas 2 e 3 , onde foram liberados aproximadamente 270 e 540 insetos/gaiola, em média, índices até de $86,7 \%$ e $85,7 \%$ de parasitismo respectivamente (Tabela 1 ).

Tabela 1. Efeito da liberação de parasitóides $A$. citricola no controle de P. citrella em citros. Porcentagem de larvas parasitadas em cada época de avaliação. Álvares Machado, SP (Ano 2000).

\begin{tabular}{|c|c|c|c|c|c|}
\hline \multirow{2}{*}{ Tratamento } & \multirow{2}{*}{$\begin{array}{l}\text { Parasitóides } \\
\text { emergidos }\end{array}$} & \multicolumn{4}{|c|}{ Emergência após a liberação (\%)* } \\
\hline & & 23 dias & 40 dias & 58 dias & 70 dias \\
\hline \multirow{2}{*}{ Parcela 1} & Ageniaspis citricola & $5,08 \pm 1,57$ & $3,70 \pm 0,67$ & $6,25 \pm 1,05$ & $10,40 \pm 0,38$ \\
\hline & Parasitóides nativos & $14,28 \pm 1,17$ & $33,33 \pm 1,75$ & $18,75 \pm 1,59$ & $15,79 \pm 1,48$ \\
\hline \multirow{2}{*}{ Parcela 2} & Ageniaspis cirricola & $6,25 \pm 0,87$ & $0,00 \pm 0,01$ & $16,67 \pm 0,96$ & $86,67 \pm 2,99$ \\
\hline & Parasitóides nativos & $12,50 \pm 1,84$ & $40,91 \pm 1,91$ & $83,33 \pm 7,04$ & $0,00 \pm 0,01$ \\
\hline \multirow{2}{*}{ Parcela 3} & Ageriiaspis citricola & $0,00 \pm 0,01$ & $1,69 \pm 0,36$ & $0,00 \pm 0,01$ & $85,70 \pm 11,30$ \\
\hline & Parasitóides nativos & $0,00 \pm 0,01$ & $16,95 \pm 1,39$ & $12,50 \pm 2,82$ & $0,00 \pm 0,0 \mathrm{I}$ \\
\hline
\end{tabular}

* Média de quatro repetições, seguidas do desvio padrão (SD).

Na parcela 2, a incidência do parasitóide $A$. citricola aumentou de $6,25 \%$, com 23 dias após a liberação, para 86,67\%, aos 73 dias, evidenciando o estabelecimento da espécie na área; ao mesmo tempo se observou a incidência de parasitóides nativos evoluir de $12,50 \%$ aos 23 dias após a liberação, para $83,33 \%$ aos 58 dias. Aos 73 dias nenhum parasitóide nativo foi detectado na amostragem, evidenciando que, à medida que o parasitóide introduzido se estabelece na região, os parasitóides nativos tendem a pro- 
curar hospedeiros alternativos. Este mesmo fato já observado por Pomerinke \& Stansly (1998) e Diez et al. (2000).

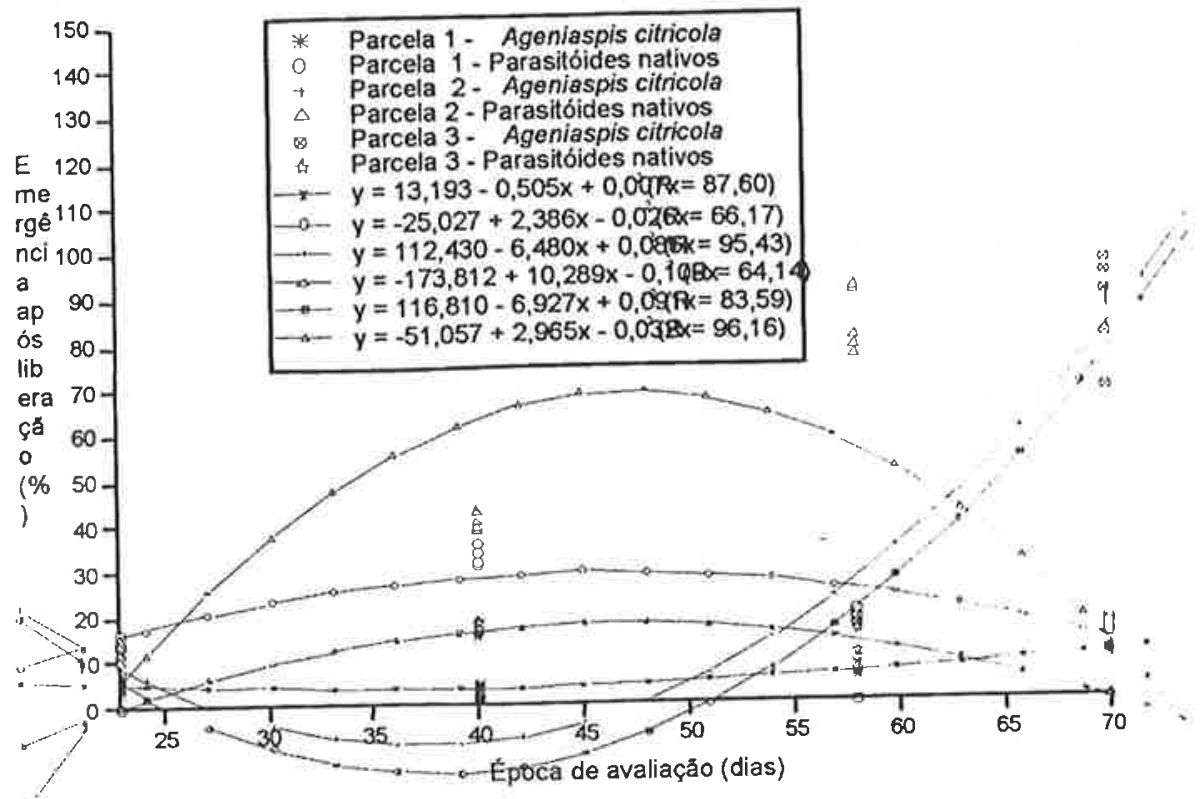

Figura 1. Regressão estabelecida entre emergência de parasitóides após liberação do parasitóide $A$. citricola e épocas de avaliação.

$\mathrm{Na}$ regressão estabelecida entre as épocas de avaliação e a emergência dos parasitóides após liberação, observou-se equivalência entre os 60-65 dias após a liberação, em que tanto o parasitóide exótico liberado na área, $A$. citricola, quanto os parasitóides nativos, apresentaram alto indice de parasitismo de P. citrella (Figura 1).

$\mathrm{Na}$ parcela 3, a incidência do parasitóide $A$. citricola aumentou de $1,69 \%$, aos 40 dias, para $85,71 \%$ com 73 dias, enquanto que a incidência de parasitóides nativos decresceu de 16,95\% (40 dias) para zero aos 73 dias.

As espécies de parasitóides nativos ocorridos foram Galeopsomyia sp. e Cirrospihus sp. ambos de família Eulophidae, e Elasmus sp., da família Elasmidae. 
Pode-se observar que, à medida que o parasitóide introduzido $A$. citricola se estabeleceu na área de liberação, o parasitismo por parasitóides nativos diminuiu, provavelmente devido à agressividade característica deste espécie de parasitóide, que apresenta poliembronia, isto é, de cada indivíduo parasitado em média nascem 3 novos indivíduos (Gravena, 2000).

A realização de liberação em pomares deve ser sincronizada com a emissão de fluxos de brotação nova pela planta e alta infestação da praga, além de se observar a utilização de produtos seletivos na condução da cultura, para uma melhor eficiência no estabelecimento do parasitóide na área.

\section{REFERENCIAS BIBLIOGRAFICAS}

BROWNING, H.; PEÑA, J.E., 1995. Biological Control of the Citrus Leafminer by its Native Parasitoids and Predators. Citrus Industry, 4(76):46-48.

CHAGAS, M.C.M.; NAMEKATA, T; ROSSI, A.C.; CERÁVOLO, L.C.; MONTES, S.M.N.M.; PARRA, J.R.P., 1998. Papel da Lagarta Minadora dos Citros Phyllocnistis citrella (Lepidoptera, Gracillariidae) na Infecção pelo Cancro Cítrico. In: CONGRESSO BRASILEIRO DE ENTOMOLOGIA, 17., Rio de Janeiro. Resumos. p.336. DIEZ, P.A.; FRIAS, E.A.; OVRUSKU, N.E.; FIDALGO, A.A., 2000.

Preliminary Evaluation of Ageniaspis citricola (Hym: Encyrtidae) Attacking Phyllocnistis citrella (Citrus Leafminer) in Argentina. In: INTERNATIONAL CONGRESS OF ENTOMOLOGY, 21., Foz do Iguaçu. Abstracts. p.383.

FUNDECITRUS, 2001. Inimigo Natural Reduz Larva Minadora. Revista do Fundecitrus, 103:68.

GRAVENA, S., 1998. Galeopsomyia fausta, nosso Parasito da Larva Minadora. Informativo Agropecuário: Coopercitrus, 140:28.

GRAVENA, S., 2000. Inseticidas Reduzem a Eficácia da Vespinha Ageniaspis! Revista do Fundecitrus, 171:17.

HEPPNER, J.B., 1993. Citrus Leafminer, Phyllocnistis citrella, in Florida (Lepidoptera: Gracillariidae: Phyllocnistinae). Tropical Lepidoptera, 4(1):49-64. 
KNAPP, J.L.; ALBRIGO, L.G; BROWING, H.W.; BULLOCK, R.C.; HEPPNER, J.B.; HALL, D.G; HOY, M.A.; NGUYEN, R. PEÑA, J.E.; STANSLY, P.A., 1995. Citrus Leafminer, Phyllocnistis citrella Stainton : Current Status in Florida. Gainsville: Florida Cooperative Extensive Service - Intituto of Food and Agricultural Sciences, University of Florida, Gainesville, 35p.

NASCIMENTO, A.S.; VIDAL, C.A., 1998. Ocorrência de Parasitóides e Local de Preferência para Desenvolvimento da Larva Minadora das Folhas dos Citros Phyllocnistis citrella Stainton, 1856, em Cruz das Almas, BA. In: CONGRESSO BRASILEIRO DE ENTOMOLOGIA, 17., Rio de Janeiro. Resumos. p.324.

PAIVA, P.E.B.; PENTEADO-DIAS, M.A ; BENVENGA, S.R.; GRAVENA, R.; GRAVENA, S., 1998. Galeopsomyia fausta: Principal Parasitóide Nativo da Minadora das Folhas dos Citros em São Paulo. In: CONGRESSO BRASILEIRO DE ENTOMOLOGIA, 17. Rio de Janeiro. Resumos. p.373.

PENTEADO-DIAS, A.M.; GRAVENA, S.; PAIVA, P.E.B.; PINTO, R.A., 1997. Parasitóides de Phyllocnistis citrella Stainton (Lepidoptera: Gracillariidae: Phyllocnistinae) no Estado de São Paulo. Laranja, 18(1):79-84.

POMERINKE, M.A.; STANSLY, P.A., 1998. Establishement of Ageniaspis citricola (Hymenoptera: Encyrtidae) for Biological Control of Phyllocnistis citrella (Lepidoptera: Gracillariidae) in Flórida. Flórida Entomologist, 81(3):361-72.

SMITH, J.M.; HOY, M.A., 1995. Rearing Methods for Ageniaspis citricola (Hymenoptera: Encyrtidae) Released in a Classical Biological Control Program for the Citrus Leafminer, Phyllocnistis citrella (Lepidoptera: Gracillariidae), Florida Entomologist, 78(4):600-608. 